\title{
Optimal Power Factor Regulation of Dispersed Wind Farms under Diverse Load and Stochastic Wind Conditions Based on Improved Firefly Algorithm
}

\author{
Hao Liu (D), ${ }^{1}$ Wei Wang, ${ }^{1}$ Jia Cui, ${ }^{2}$ and Fen Tang ${ }^{1}$ \\ ${ }^{1}$ National Active Distribution Network Technology Research Center, Beijing Jiaotong University, Beijing, 100044, China \\ ${ }^{2}$ School of Electrical Engineering, Shenyang University of Technology, Shenyang, 110870, China \\ Correspondence should be addressed to Hao Liu; 1120882185@qq.com
}

Received 22 July 2018; Accepted 13 September 2018; Published 27 September 2018

Academic Editor: Ramón I. Diego

Copyright (C) 2018 Hao Liu et al. This is an open access article distributed under the Creative Commons Attribution License, which permits unrestricted use, distribution, and reproduction in any medium, provided the original work is properly cited.

\begin{abstract}
Aiming at the instability of voltage and large network loss of dispersed wind farms (DWFs) integration into distribution network (DN), an optimal power factor regulation method based on improved firefly algorithm is proposed. Firstly, the generalized load model based on the static voltage characteristics is analyzed. Then reactive power capabilities of DWFs are thoroughly discussed and the influences of DWFs with variable power factor on network loss and voltage profile are presented. In order to reduce network loss and enhance power quality, optimal power factor regulation of DWFs based on improved firefly algorithm (IFA) is proposed. Finally, results of the benchmark IEEE-33 node system show the feasibilities and validities of the proposed method and the superiorities of proposed IFA are revealed by comparison with the existing algorithms.
\end{abstract}

\section{Introduction}

As the development of distributed energy, dispersed wind farms (DWFs) are expected to comprise a significant portion of future power generation. However, high penetration level of DWFs integration into the distribution network (DN) presents major challenges in terms of planning and operation of the power grid. According to traditional "fit and forget" practice, DWFs usually operate at maximum power point and unity power factor, which are mostly treated as negative fluctuant loads in the conventional DWF management $[1$, 2]. In this way, the inherent uncertainty and variability of wind power supply further deteriorate the voltage profiles of $\mathrm{DN}$. To facilitate the safe and stable operation of DN, the penetration level is limited. Otherwise additional reactive power compensation equipment is needed, increasing the investment and operation cost. Actually, DWFs are connected to DN through their interfacing converters. Such power electronics based DWFs have great potentials in power regulation capabilities, which could be considered in the operation process. In this way, DWFs take some degree of responsibility for system support and their roles are changed from the passive actuators into active participators, which is an important element of changing traditional DN operation into active DN operation $[3,4]$.

To facilitate an active DN operation, how to reestablish and resolve system optimization function with the introduction of such active participators is essential. On one hand, operation related problems mostly focus on the optimization of typical indexes under a single load type condition in the literature [5-7]. However, most of the DWFs are installed near end-users for the purpose of meeting on-site power demand. The loads are not explicitly single type such as constant power, industrial, commercial, and residential loads but rather the combination of these types in the practical applications $[8,9]$. Such loads have different influences on the voltage profile of DN. Thus, load types might not be neglected in the operation of DWFs especially considering reactive power support capabilities.

On the other hand, various optimization techniques have been suggested in recent decades. The solution techniques could be classified into conventional, intelligent searches, and fuzzy set applications. Compared to other intelligent algorithms, the firefly algorithm is faster and the variable 


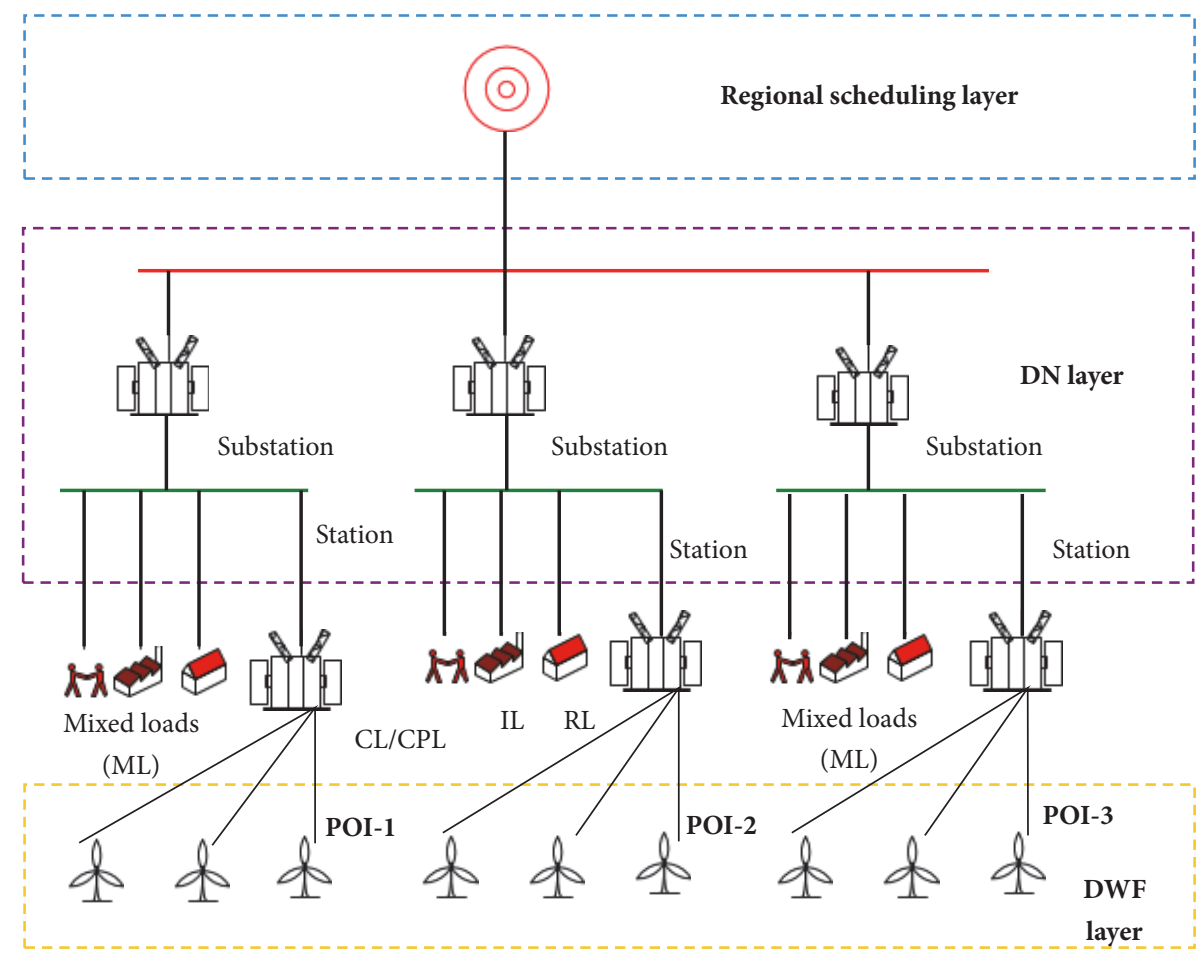

FIgure 1: Typical topology of DWFs in DN.

parameter adjustment is simplified. But the common disadvantages of these algorithms are premature convergence of the population and slow convergence rate $[10,11]$.

To address these issues, optimal operation of DWFs considering reactive power support capabilities and load types is proposed for network loss reduction and power quality enhancement. In this paper, the generalized load model based on the static voltage characteristics is first introduced. Afterwards, the reactive power capabilities of DWFs are thoroughly discussed. On this basis, the multiobjective optimization function is established, considering the various load and stochastic wind conditions as well as the system equality and inequality constraints. Furthermore, the improved firefly algorithm (IFA) is introduced to solve the given optimization function. Finally, results in the benchmark IEEE-33 node radial distribution system validate the effectiveness of the proposed method.

\section{Generalized Load Model}

The typical topology of DWFs in distributed network system is shown in Figure 1, where DWFs are integrated at point of interconnection (POI) and near diverse end-users. The loads are not explicitly single type such as constant power (CPL), industrial (IL), commercial (CL), and residential loads (RL). Depending on the nature of area, we defined the mixed load (ML) which combines different kinds of loads. Since different loads correspond to the different characteristics of network loss and voltage profile, the loads' characteristics need to be analyzed. Therefore, a generalized load model relating the load power to voltage should be investigated. Based on
TABLE 1: Voltage characteristic coefficients for different load types.

\begin{tabular}{lcccccc}
\hline \multirow{2}{*}{ Season } & \multicolumn{2}{c}{$\mathrm{RL}$} & \multicolumn{2}{c}{$\mathrm{CL}$} & \multicolumn{2}{c}{$\mathrm{IL}$} \\
& $\alpha_{1}$ & $\beta_{1}$ & $\alpha_{2}$ & $\beta_{2}$ & $\alpha_{3}$ & $\beta_{3}$ \\
\hline Spring & 1.2 & 4.38 & 1.26 & 3.35 & 0.18 & 6 \\
Summer & 0.72 & 2.96 & 1.26 & 3.5 & 0.18 & 6 \\
Autumn & 0.98 & 3.52 & 0.98 & 3.95 & 0.18 & 6 \\
Winter & 1.04 & 4.18 & 1.5 & 3.15 & 0.18 & 6 \\
\hline
\end{tabular}

the static voltage characteristics, the generalized load model could be given as [12]

$$
\begin{gathered}
P_{\mathrm{Li}}=P_{L i}^{D}\left(\begin{array}{c}
a_{1}\left(\frac{U_{i}}{U_{N}}\right)^{\alpha_{0}}+b_{1}\left(\frac{U_{i}}{U_{N}}\right)^{\alpha_{1}} \\
+c_{1}\left(\frac{U_{i}}{U_{N}}\right)^{\alpha_{2}}+d_{1}\left(\frac{U_{i}}{U_{N}}\right)^{\alpha_{3}}
\end{array}\right) \\
Q_{\mathrm{Li}}=Q_{L i}^{D}\left(\begin{array}{c}
a_{2}\left(\frac{U_{i}}{U_{N}}\right)^{\beta_{0}}+b_{2}\left(\frac{U_{i}}{U_{N}}\right)^{\beta_{1}} \\
+c_{2}\left(\frac{U_{i}}{U_{N}}\right)^{\beta_{2}}+d_{2}\left(\frac{U_{i}}{U_{N}}\right)^{\beta_{3}}
\end{array}\right)
\end{gathered}
$$

where $U_{N}$ and $U_{i}$ are, respectively, the nominal and actual node voltages, $P_{L i}, Q_{L i}, P_{L i}{ }^{D}$, and $Q_{L i}{ }^{D}$, are, respectively, the load active power and reactive power under nominal and actual node voltage conditions.

In (1), load demands of different types are described by the exponent and weight values [13]. The typical exponent values of different load types are shown in Table 1. Obviously, the exponent values $\alpha_{0}$ and $\beta_{0}$ are both zero for the constant 


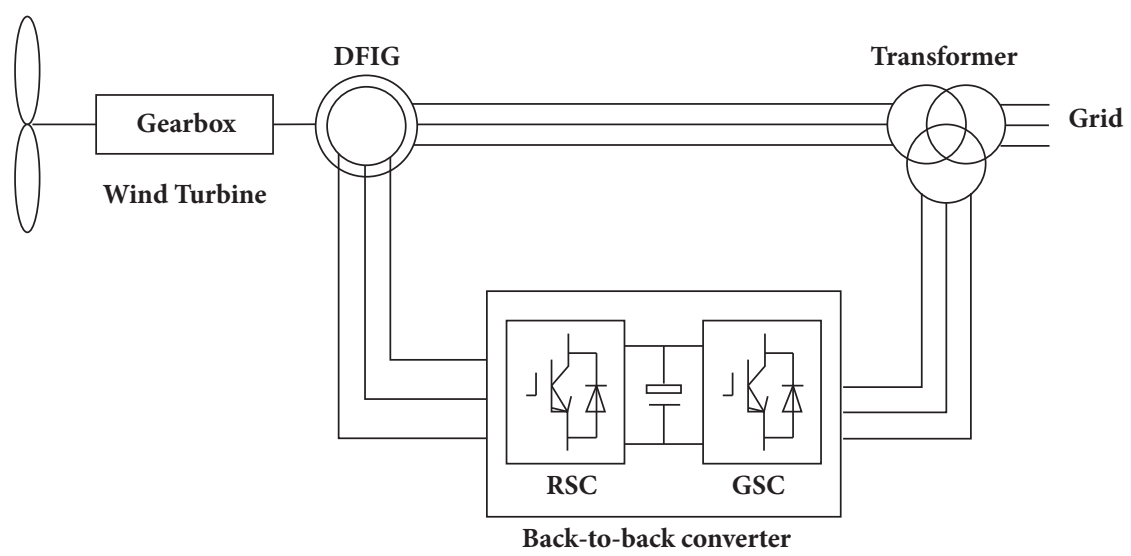

FIGURE 2: DFIG based wind generator.

power load. $a_{1}, b_{1}, c_{1}$, and $d_{1}$ are the active power weight coefficients, $a_{2}, b_{2}, c_{2}$, and $d_{2}$ are the reactive power weight coefficients, which are determined by the percentages of active and reactive power consumption or demand.

\section{Reactive Power Support Capabilities of DWFs}

Doubly-Fed Induction Generator (DFIG) based wind generator is widely used in DWFs, which mainly consists of a wind turbine, a gearbox, DFIG, and back-to-back converters, as shown in Figure 2.

The back-to-back converters interfacing the DFIG based wind turbines to the grid play a significant role in the whole system, which are composed of grid-side converter (GSC) and rotor-side converter (RSC). The GSC is connected to the grid and is usually controlled to provide a steady DC-link voltage and meet the power quality requirements, while the RSC is connected to the rotor windings of the DFIG and control the active and reactive power injected into the grid. The total generated power is divided into the power from the stator and the power from the rotor controlled by the backto-back converter. Therefore, their reactive power support capabilities are complicated since they could be generated by both stator and rotor sides via back-to-back converters, which are thoroughly discussed in this Section [14].

By using stator voltage oriented vector control [15], due to the flux lags the voltage $90^{\circ}$ in stator side in steady state, the grid-voltage $u_{s}$ has no component on the $\mathrm{d}$ axis, which is the direct component of the stator voltage $u_{\mathrm{ds}}=0$. Finally, active power $P_{s}$ and reactive power $Q_{s}$ provided by stator side are described as

$$
\begin{aligned}
P_{s} & =\frac{3}{2} u_{q s} i_{q s}=\frac{3}{2} \frac{L_{m}}{L_{s}} u_{s} i_{q r} \\
Q_{s} & =\frac{3}{2} u_{q s} i_{d s}=-\frac{3}{2} \frac{L_{m}}{L_{s}} u_{s} i_{d r}-\frac{3}{2} \frac{u_{s}^{2}}{\omega_{s} L_{s}}
\end{aligned}
$$

where $u_{\mathrm{qs}}, i_{\mathrm{ds}}, i_{\mathrm{qs}}, i_{\mathrm{dr}}$, and $i_{\mathrm{qr}}$ are, respectively, quadrature component of the stator voltage, stator current, and rotor current in the rotating frame, $\omega_{\mathrm{s}}$ is the air-gap magnetic field rotating angular velocity of stator, $L_{\mathrm{s}}$ and $L_{\mathrm{m}}$ are, respectively, stator winding inductance and mutual inductance between stator and rotor windings, and $u_{s}$ is the peak value of stator voltage.

Therefore, the total active power $P_{\mathrm{e}}$ injected by DFIG into the grid could be derived as

$$
P_{e}=(1+s) P_{s}
$$

where $s$ is the slip.

The maximum and minimum reactive power $Q_{s}{ }^{\max }$ and $Q_{s}{ }^{\text {min }}$ provided by the stator side are derived as [16].

$$
\begin{aligned}
& Q_{s}^{\max }=-\frac{3 u_{s}^{2}}{2 \omega_{s} L_{s}}+\sqrt{\left(\frac{3 u_{s} L_{m} i_{r}^{\max }}{2 L_{s}}\right)^{2}-P_{s}^{2}} \\
& Q_{s}{ }^{\min }=-\frac{3 u_{s}^{2}}{2 \omega_{s} L_{s}}-\sqrt{\left(\frac{3 u_{s} L_{m} i_{r}^{\max }}{2 L_{s}}\right)^{2}-P_{s}^{2}}
\end{aligned}
$$

where $i_{\mathrm{r}}{ }^{\max }$ is the rotor current limit.

Similarly, the maximum and minimum reactive power $Q_{g}{ }^{\max }$ and $Q_{g}{ }^{\min }$ provided by GSC are described as

$$
\begin{aligned}
Q_{g}^{\max } & =\sqrt{S_{c}^{2}-\left(s P_{s}\right)^{2}} \\
Q_{g}^{\min } & =-Q_{g}^{\max }
\end{aligned}
$$

where $S_{\mathrm{c}}$ is the apparent power of GSC.

Combining (4) and (5), the total maximum and minimum reactive power $Q_{e}{ }^{\max }$ and $Q_{e}{ }^{\min }$ of DFIG considering both stator and rotor sides are obtained as

$$
\begin{gathered}
Q_{e}^{\max }=Q_{s}^{\max }+Q_{g}^{\max } \\
Q_{e}^{\min }=Q_{s}^{\min }+Q_{g}^{\min }
\end{gathered}
$$

It can be seen from (4) that the maximum and minimum reactive power capabilities $Q_{s}{ }^{\max }$ and $Q_{s}{ }^{\min }$ are unbalanced and the lower one dominates the overall reactive power capabilities, which could be improved by installing capacitors at the grid-connected point. Consequently, the balanced 


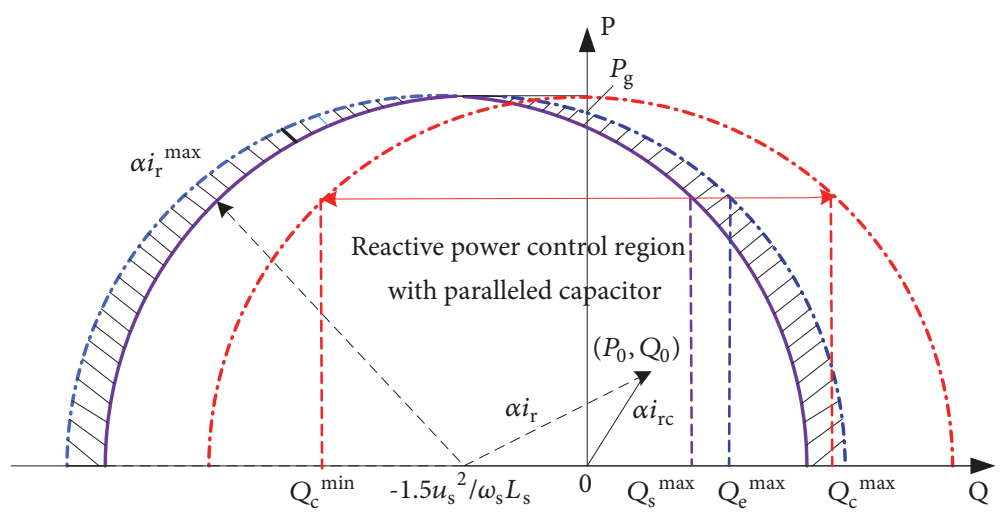

FIGURE 3: Reactive power capabilities of DFIG.

reactive power capabilities $Q_{c}{ }^{\max }$ and $Q_{c}{ }^{\min }$ with proper capacitors are revised as follows:

$$
\begin{aligned}
& Q_{c}^{\max }=\sqrt{\left(\alpha i_{r}{ }^{\max }\right)^{2}-P_{s}^{2}}+\sqrt{S_{c}{ }^{2}-\left(s P_{s}\right)^{2}} \\
& Q_{c}^{\min }=-Q_{c}^{\max }
\end{aligned}
$$

where $\alpha=1.5 u_{s} L_{m} / L_{s}$.

Figure 3 summarizes the reactive power capabilities, showing that DFIG has the great potentials in power regulation capabilities. In Figure 3, the purple solid line is the reactive power region generated by stator side and the blue dash line is the reactive power region generated by both stator and rotor sides and the shading part denoting the reactive power region of GSC. The red dash line is the reactive power region considering the added capacitor. Obviously, it has $Q_{c}{ }^{\max }>$ $Q_{e}{ }^{\max }>Q_{s}{ }^{\max }$ and the rotor current $i_{r c}$ with paralleled capacitor is much smaller than $i_{r}$ at the operating point $\left(P_{0}\right.$, $\left.Q_{0}\right)$, which means that the reactive power capabilities could be enhanced by implementing proper control and capacitors. Furthermore, with the increasing of active power, reactive power capabilities are decreased gradually, which are mainly restricted by $i_{r}^{\max }$.

\section{Influences on Network Loss and Voltage Profile with the Integration of DWFs}

4.1. Influence on Network Loss. In an M-node distribution network, the total active and reactive power network loss $P_{L}$ and $Q_{L}$ could be expressed as [17]

$$
\begin{aligned}
P_{L} & =\sum_{i=1}^{M} \sum_{j=1}^{M}\left[k_{i j}\left(P_{i} P_{j}+Q_{i} Q_{j}\right)+h_{i j}\left(Q_{i} P_{j}-P_{i} Q_{j}\right)\right] \\
Q_{L} & =\sum_{i=1}^{M} \sum_{j=1}^{M}\left[\gamma_{i j}\left(P_{i} P_{j}+Q_{i} Q_{j}\right)+\xi_{i j}\left(Q_{i} P_{j}-P_{i} Q_{j}\right)\right]
\end{aligned}
$$

where

$$
\begin{aligned}
k_{i j} & =\frac{r_{i j}}{U_{i} U_{j}} \cos \left(\delta_{i j}\right) \\
h_{i j} & =\frac{r_{i j}}{U_{i} U_{j}} \sin \left(\delta_{i j}\right) \\
\gamma_{i j} & =\frac{x_{i j}}{U_{i} U_{j}} \cos \left(\delta_{i j}\right) \\
\xi_{i j} & =\frac{r_{i j}}{U_{i} U_{j}} \sin \left(\delta_{i j}\right)
\end{aligned}
$$

In (8) and (9), $P_{i}, P_{j}, Q_{i}$, and $Q_{j}$ are the active and reactive power injections at the ith and jth nodes, respectively; $U_{i}$ and $U_{j}$ are the $i^{\text {th }}$ and $j^{\text {th }}$ node voltages, $\delta_{i j}$ is power angle difference between nodes $i$ and $j$, and $r_{i j}$ and $x_{i j}$ are resistance and reactance between nodes $i$ and $j$.

When the DWFs are integrated into the M-node distribution network, the total active and reactive power network loss are updated as (10), where the notations of total network loss are, respectively, modified as $P_{L D W F}$ and $Q_{L D W F}$.

$$
\begin{gathered}
P_{\mathrm{LDWF}}=\sum_{i=1}^{M} \sum_{j=1}^{M}\left[k_{i j}\left(\left(P_{\mathrm{Gi}}+P_{\mathrm{ei}}-P_{\mathrm{Li}}\right) P_{\mathrm{j}}+Q_{\mathrm{j}}\left(Q_{\mathrm{Gi}}+\tan \left(\phi_{i}\right) P_{\mathrm{ei}}-Q_{\mathrm{Li}}\right)\right)\right. \\
\left.+h_{i j}\left(\left(Q_{\mathrm{Gi}}+\tan \left(\phi_{i}\right) P_{\mathrm{ei}}-Q_{\mathrm{Li}}\right) P_{\mathrm{j}}-\left(P_{\mathrm{Gi}}+P_{\mathrm{ei}}-P_{\mathrm{Li}}\right) Q_{\mathrm{j}}\right)\right] \\
Q_{\mathrm{LDWF}}=\sum_{i=1}^{M} \sum_{j=1}^{M}\left[\gamma_{i j}\left(\left(P_{\mathrm{Gi}}+P_{\mathrm{ei}}-P_{\mathrm{Li}}\right) P_{\mathrm{j}}+Q_{\mathrm{j}}\left(Q_{\mathrm{Gi}}+\tan \left(\phi_{i}\right) P_{\mathrm{ei}}-\mathrm{Q}_{\mathrm{Li}}\right)\right)\right. \\
\left.+\xi_{i j}\left(Q_{\mathrm{Gi}}+\left(\tan \left(\phi_{i}\right) P_{\mathrm{ei}}-\mathrm{Q}_{\mathrm{Li}}\right) P_{\mathrm{j}}-\left(P_{\mathrm{Gi}}+P_{\mathrm{ei}}-P_{\mathrm{Li}}\right) Q_{\mathrm{j}}\right)\right]
\end{gathered}
$$




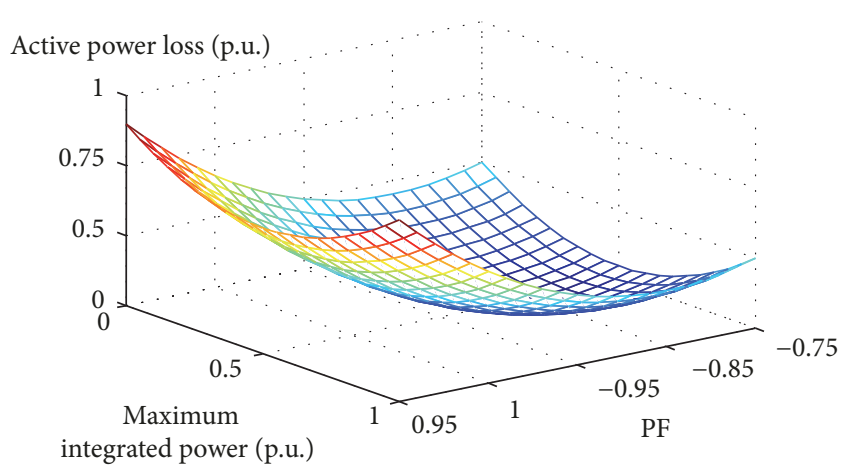

FIGURE 4: Impacts of maximum integrated power and PF on network loss.

In (10), $P_{G i}$ and $Q_{G i}$ are the injecting active and reactive power from grid at node $i ; P_{e i}$ (see (3)) and $\phi_{i}$ are the injecting active power and power factor angle of DWF at node $i$; $P_{L i}$ and $Q_{L i}$ (see (1)) are load active and reactive power at node $i$, respectively.

Indexes of active and reactive power network loss APL and RPL could be further derived as

$$
\begin{aligned}
& \eta_{A P L}=\frac{P_{L D W F}}{P_{L}} \\
& \eta_{R P L}=\frac{Q_{L D W F}}{Q_{L}}
\end{aligned}
$$

Obviously, the active power and power factor (PF) of DWFs have great influences on network loss. In this paper, negative power factor represents capacitive reactive power while positive power factor is on behalf of inductive reactive power according to the convention. For a given PF, the network loss will first decrease and then increase with the increase of the integrated power as shown in Figure 4. For a given integrated power, the change trend is similar. That means that the curve which relates network loss to integrated active power and power factor presents parabolic shape. As maximum integrated power of DWF increasing, the power from upstream branch to load will be reduced, resulting in decreased active power loss. After the minimum network loss is achieved, if maximum integrated power continues to increase, the reverse power flow will be shown up, which increases the network loss as illustrated in Figure 4. It implies that the system performance could be optimized through power factor regulation of DWFs for a given distribution network.

4.2. Influence on Voltage Profile. Compared to the conventional $\mathrm{DN}$, the voltage profile is quite different with the introduction of both the diverse load types and stochastic wind power. In traditional $\mathrm{DN}$, the voltage profile is simple. The node voltage usually decreases as the electrical distance from the node to the main utility increases and may become lower than the minimum voltage at the end of the feeder. However, with the integration of DWFs, the voltage profile is complicated. Three DWFs were connected into IEEE 33 system, located in the points 14,24 , and 30 , and the voltage curves are shown in Figure 5.

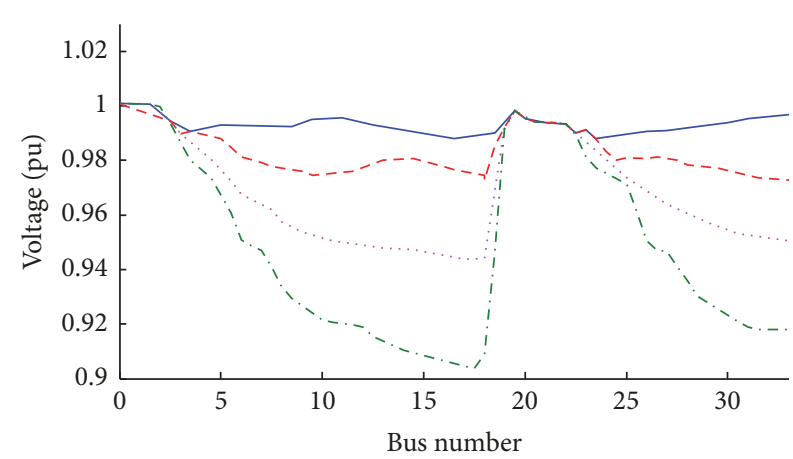

$$
\begin{aligned}
& \stackrel{P F}{ } 0.95 \\
& ---1 \\
& \cdots \cdots-0.95 \\
& - \text { - - }-0.85
\end{aligned}
$$

FIgURE 5: 33-bus network voltage profile in the four PF scenarios.

It can be concluded that the PF can make a big difference on the voltage profile. If power factor or reactive power of DWFs can be adjusted, the voltage profile may be improved. The comparative results also indicate that constant fixed power factor values are not always the preferred solutions.

\section{Optimal Power Factor Regulation of DWFs}

Based on the above analysis, we can know the network loss and voltage profile can be further optimized by adjusting PF.

\subsection{Multiobjective Optimization Function}

5.1.1. Multiobjective Optimization Function. In order to get the optimal results, considering various load types and stochastic wind conditions, multiobjective optimal function is constructed, whose equation is

$$
M O F=\min \left(k_{1} \eta_{A P L}+k_{2} \eta_{R P L}+k_{3} \delta_{v}\right)
$$

where $\eta_{A P L}$ is the degree of active power loss, $\eta_{R P L}$ means the degree of reactive power loss, and $\delta_{v}$ is voltage deviation, whose calculation is

$$
\delta_{v}=\max _{j=1}^{M}\left(\frac{U_{N}-U_{j}}{U_{N}}\right)
$$

Form the above equations, it can be known that the network loss and voltage deviation are considered to construct MOF. The optimal value is the minimum MOF. Besides, all weight factors in (12) are determined according to the individual impacts and importance of the particular index. The main objective is to minimize the overall network loss of system, so $\eta_{A P L}$ gets the highest weight of 0.45 and $\eta_{R P L}$ gets the second highest weight of 0.38 , while $\delta_{\mathrm{v}}$ gets a weight of 0.17 in order to maintain good power quality and voltage profile.

5.2. Constraints. The constraints should be set to solve the equations, which include power flow, output power, node 
voltage, branch current, and climbing rate. The details are shown as follow:

(1) Power flow equality constraints

$$
\begin{aligned}
& P_{\mathrm{Gi}}+P_{\mathrm{ei}}-P_{\mathrm{Li}}-U_{i} \sum_{j=1}^{M} U_{j}\left(G_{i j} \cos \delta_{i j}+B_{i j} \sin \delta_{i j}\right)=0 \\
& Q_{\mathrm{Gi}}+\tan \left(\phi_{i}\right) P_{\mathrm{ei}}-Q_{\mathrm{Li}} \\
& -U_{i} \sum_{j=1}^{M} U_{j}\left(G_{i j} \cos \delta_{i j}-B_{i j} \sin \delta_{i j}\right)=0
\end{aligned}
$$

where $B_{i j}$ and $G_{i j}$ are, respectively, susceptance and conductance between nodes $i$ and $j, P_{G i}$ and $Q_{G i}$ are, respectively, injected active and reactive power from grid, $P_{e i}$ and $Q_{e i}$ are the active and reactive power of DWF at node $i, U_{i}^{\min }$ and $U_{i}^{\max }$ are, respectively, voltage lower and upper limits, and $U_{1}$ is the first node voltage.

(2) Output power inequality constraints of DWFs are

$$
\begin{aligned}
0 & \leq P_{\text {ei }} \leq P_{D W F i}^{\max } \\
\left|Q_{e i}\right| & =\left|\tan \left(\phi_{i}\right) P_{\text {ei }}\right| \leq Q_{c}{ }^{\max }
\end{aligned}
$$

where $P_{D W F i}{ }^{\max }$ is the real-time maximum power according to the real-time wind conditions and the maximum balanced reactive power $Q_{c}^{\max }$ is determined according to (7), fully considering the reactive capability of the DWFs with shunt capacitor.

(3) Node voltage inequality constraints are

$$
U_{i}^{\min } \leq U_{i} \leq U_{i}^{\max }
$$

(4) Node voltage inequality constraints considering no reverse power flow at the first node are

$$
U_{i} \leq U_{1}
$$

(5) Branch current inequality constraints are

$$
I_{i} \leq I_{i}^{\max }
$$

Here $I_{i}^{\text {max }}$ is the $i^{\text {th }}$ branch current upper limit.

(6) Climbing rate constraints of DWFs

$$
\Delta P_{D W F i}^{\min } \leq P_{e i, t}-P_{e i, t-1} \leq \Delta P_{D W F i}^{\max }
$$

In (19), $\Delta P_{D W F i}^{\text {min }}$ and $\Delta P_{D W F i}{ }^{\max }$ are, respectively, declining and rising rate limits of DWFs.

\subsection{Improved Firefly Algorithm Optimization}

5.3.1. Firefly Algorithm Theories. The conventional Firefly algorithm (FA) is a metaheuristic proposed by Xin-She Yang and inspired by the flashing behaviour of fireflies. FA has many advantages, such as simple operation, strong robustness, easy implementation, etc. compared with the existing genetic algorithm and particle swarm optimization algorithm, FA owns better ability to search the global optimal solution, what's more, whose convergence speed is faster. FA has been successfully applied to the optimization of nonlinear issues [18]. The following simplified rules are considered. All fireflies are unisexual, so that any individual firefly will be attracted to all other fireflies. Attractiveness is proportional to their brightness and, for any two fireflies, the less bright one will be attracted by the brighter one. Brightness and, therefore, attractiveness decrease with the distance between the fireflies. If there are no fireflies brighter than a given firefly, it will move randomly. Mathematically, the FA can be expressed by three equations [19].

(1) Light intensity $L I$ is

$$
L I=L I_{0} \times e^{-\gamma d_{i j}}
$$

where $L I_{0}$ is the maximum light intensity at $d_{i j}=0$ and $d_{i j}$ is the Euclidean distance between two fireflies $i$ and $j$. Because of the light absorption in medium, the light intensity decreases as the mutual distance increases, which is controlled by the absorption coefficient $\gamma$.

(2) Attractiveness $\beta$ is

$$
\beta=\beta_{0} \times e^{-\gamma d_{i j}^{2}}
$$

where $\beta_{0}$ is the maximum attractiveness.

(3) When firefly $i$ is attracted by firefly $j$, the update movement equation can be described as

$$
x_{j}=x_{i}+\beta\left(x_{i}-x_{j}\right)+\alpha(\text { rand }-0.5)
$$

where $x_{i}$ and $x_{j}$ are the positions of fireflies $i$ and $j, \alpha$ is constant and refers to the random movement factor, and rand is a random number, generated in between 0 and 1 , with uniform distribution. The brightness is determined by the value of the objective function of a given problem [20].

5.3.2. Improved Firefly Algorithm Principles. Based on its pattern, the chaos motion can traverse all states in a certain range without repeating and optimization search using chaos variables is better than blindly and disorderly random search. To avoid premature convergence of the population and slow convergence rate, improved FA (IFA) is introduced, where the chaos theory is integrated into optimization parameters of the firefly algorithm.

Equations (20) to (22) indicate that the firefly parameters $\alpha, \beta$, and $\gamma$ play significant roles in the optimization process. If these parameters can be randomly changed in the feasible region, the global optimization ability could be improved. Thus, logistic mapping function is used to regulate these parameters, the improved equations of $x_{j}, \gamma(t)$, and $\alpha(t)$ are shown in

$$
\begin{aligned}
x_{j} & =x_{i}+\beta\left(x_{i}-x_{j}\right)+\alpha(t)(\text { rand }-0.5) \\
\gamma(t) & =u_{1} \gamma(t-1)[1-\gamma(t-1)] \\
\alpha(t) & =u_{2} \alpha(t-1)[1-\alpha(t-1)]
\end{aligned}
$$

Here chaos parameter $\left[u_{1} u_{2}\right]$ is set to [4 4$]$. 


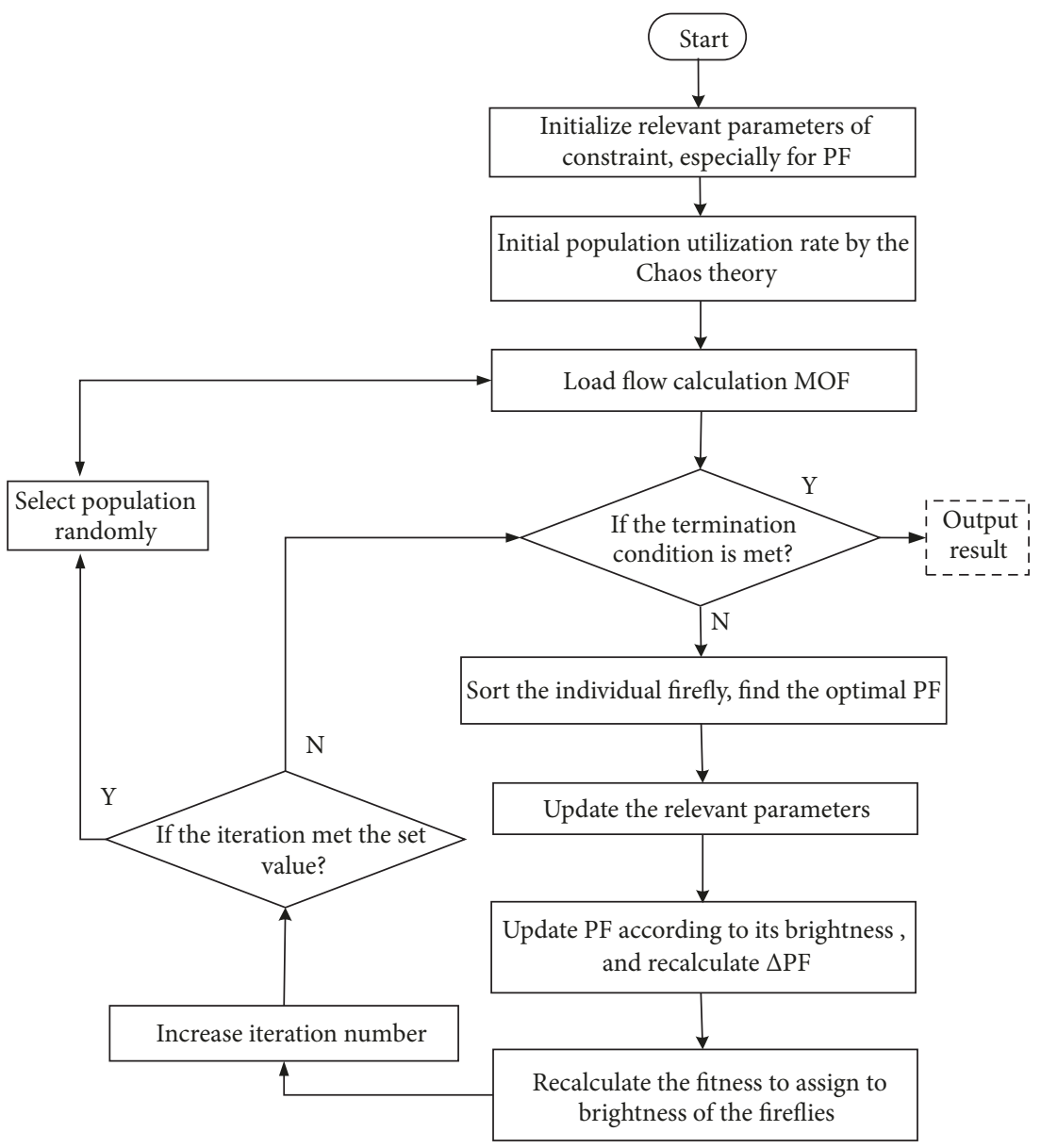

FIGURE 6: The flow chart of improved firefly algorithm.

After each iteration, a set of random parameter values $[\alpha \beta \gamma]$ are calculated by logistic mapping function. The range of three parameters is within rand $[0,1]$ in the iteration, which effectively leads the algorithm to land at the best solution with minimum computational effort and provides a good way to move away from the local search to a search on the global scale. The modified firefly could improve the convergence performance.

5.4. Optimal Power Factor Regulation of Dispersed Wind Farms Based on Improved Firefly Algorithm. The comprehensive procedures for the multiobjective optimal function using IFA are given through the flowchart of Figure 6.

In Figure 6, $\mathrm{PF}$ is the firefly position, the differences of $P F$ are $(\triangle P F)$, which means the attractiveness, the exponential function of $\triangle P F$ means the attractiveness, $L I$ is MOF, and the global best MOF is the largest $L I$.

At each iterative step, the light intensity and the attractiveness of each firefly are calculated. $L I$ of each firefly is compared with light intensity of all other fireflies and the positions of the fireflies are updated using (22). The firefly with the largest $L I$ is considered as the global optimal after completing an adequate number of iterations.

The purpose is to obtain the optimized power factor. Therefore, the position variable in (22) is actually the power factor, whereas the second and third terms of (22) can be regarded as the correction terms for power factor.

\section{Verification of Control Method and Comparison}

6.1. Verification of Optimized Control Method. The benchmark IEEE-33 node radial distribution system is used for validating the proposed optimization method as shown in Figure 7. Different load types (RL, IL, CL and ML) with the same peak active and reactive power values $3.72 \mathrm{MW}$ and 2.30 MVar are conducted, where mixed load (ML) consists of $40 \%$ industrial load (IL), 45\% residential load (RL), and 15\% commercial load (CL), respectively. Furthermore, the data with one full year from August 2014 to August 2015 in a real DWF is employed and analyzed as the basis of forecasting. The Weibull distribution is used for characterizing wind speed. Taking one-day data for test, the typical active power profiles of diverse loads and DWFs are shown in Figure 8. To find a better solution, the empirical parameter settings are listed in Table 2.

As shown in Figure 8, the active power of DWF has stronger randomness compared with other kinds of loads. Generally, RL, IL, CL, and ML have similar variation characteristics in one day. 
TABLE 2: Terminologies and parameter settings of IFA and DN system with integration of DWFs.

\begin{tabular}{lcc}
\hline Parameter & Value & Description \\
\hline Population size & 50 & Number of Fireflies/Particles \\
$N_{g}$ & 200 & Number of generations \\
$A$ & {$[0,1]$} & Randomization parameter \\
$\beta_{0}$ & 0.1 & Initial attractiveness \\
$\Gamma$ & 0.2 & Absorption coefficient \\
\hline
\end{tabular}

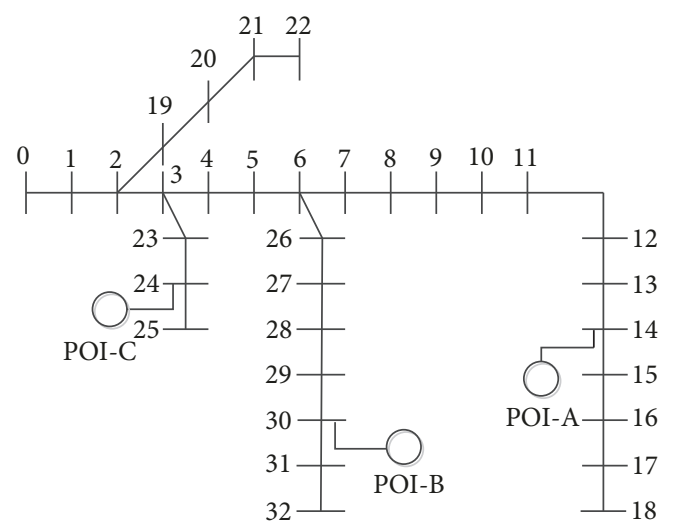

FIGURE 7: IEEE-33 node radial distribution system with 3 POIs of DWFs.

In order to demonstrate the effectiveness of network loss under diverse load type conditions by using four constant PFs (0.95, unity power factor, $-0.95,-0.85)$ and proposed optimal PF control method, respectively, both single load type and mixed load type are conducted, where three single load types (IL, RL, and CL) are with the same peak active and reactive power values $3.72 \mathrm{MW}$ and 2.30 MVar, and the mixed load type consists of $40 \%$ industrial load, $45 \%$ residential load, and $15 \%$ commercial load. The results $\eta_{\mathrm{APL}}$ and $\eta_{\mathrm{RPL}}$ of different load types are shown in Figure 9.

It can be seen in Figure 9 that $\eta_{A P L}$ and $\eta_{R P L}$ of different load types present quite differently. $\eta_{A P L}$ and $\eta_{R P L}$ for CL are the highest and the minimum values are achieved for RL. $\eta_{A P L}$ and $\eta_{R P L}$ of CL are even five times higher than those of IL, which indicates that load types might not be neglected in the optimization process. Compared to four constant PFs (0.95, unity power factor, -0.95 , and -0.85$), \eta_{A P L}$ and $\eta_{R P L}$ are achieved by using the proposed optimal PF. The results indicate that the network loss can be decreased significantly by using the proposed optimal PF method, and the effectiveness of the proposed method is proved.

To further investigate the effectiveness of the proposed method on voltage profile, one-day voltage profile is under mixed load condition by using four constant PFs ( 0.95 , unity power factor, -0.95 , and -0.85$)$, and proposed optimal PF regulations are comparatively presented in Figure 10.

It can be easily known that the voltage profile is around 1 based on the proposed optimal PF method, which is to say that the voltage deviation is around 0 and the stability is the best. Compared with the positive power factor, when the values of PFs are 0.95 and 1 , the DFWs need reactive power

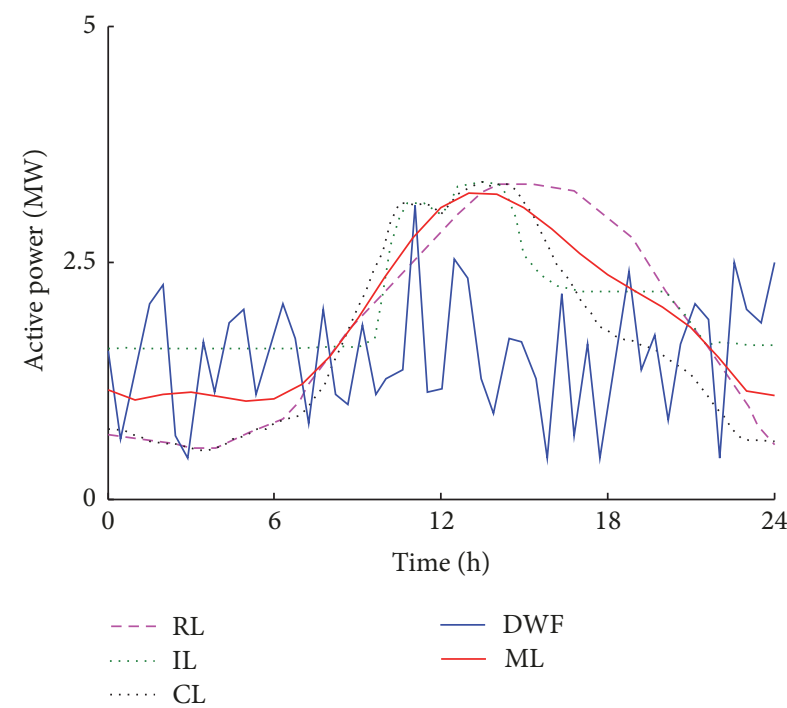

FIGURE 8: Typical active power profiles of diverse load types and DWF used.

and the voltage deviation is large $(>0.02)$. The voltage curve of Cons -0.95 is improved because the DWFs are providing reactive power and then the reactive power injected by the upstream branch will be diminished, but the difference still exists obviously. If the DWFs generate excess reactive power, the voltage profile will be also deteriorated, as demonstrated by the curve of Cons -0.85 . Anyhow, as loads are real-time changed, the constant power factor could not give a good voltage profile all the time. Figure 10(b) describes the realtime mutative characteristics, which shows that the PF can be adjusted adaptively to obtain the optimal voltage and lowest network loss.

In summary, the varieties of loads would affect the reactive power for practical conditions and the fixed power factors cannot meet the actual distribution networks. Through the optimal PF which is calculated by the IFA algorithm, the network loss and voltage stability issues can be improved significantly.

6.2. Compare with Traditional Optimized Algorithms. The output power of conventional wind farm is high in the daytime and low at night, while the dispersed wind farms are different, whose power is easily affected by surrounding environment and shows the stochastic fluctuation; the regular of output power of dispersed wind farms is similar; thus any moment can be chosen as an example, and here we chose "Time $18 \mathrm{~h}$ " as an example.

Reference [21] used Particle Swarm Optimization (PSO) algorithm, FA algorithm was introduced in [22], and Artificial Bee Colony (ABC) algorithm was used in [23]; thus we compare the above algorithms and the parameters of each optimized algorithms are based on above references. To validate the effectiveness of the given IFA, the comparative convergence results and computation time under mixed load condition at $18 \mathrm{~h}$ are compared, and the results are shown in Figures 11(a) and 11(b). 


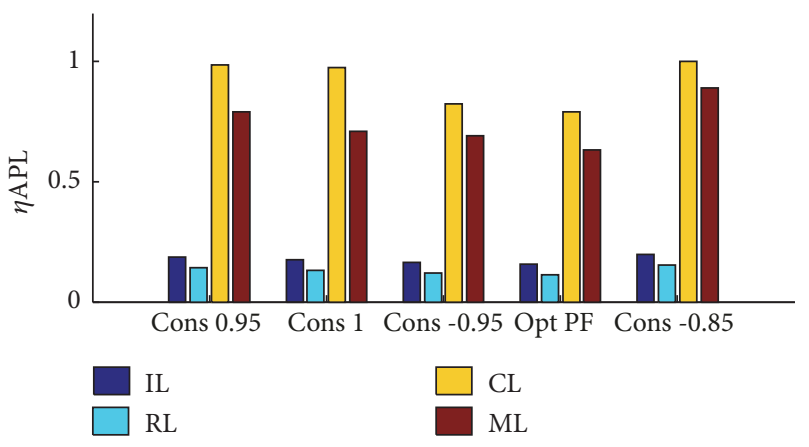

(a) APL index

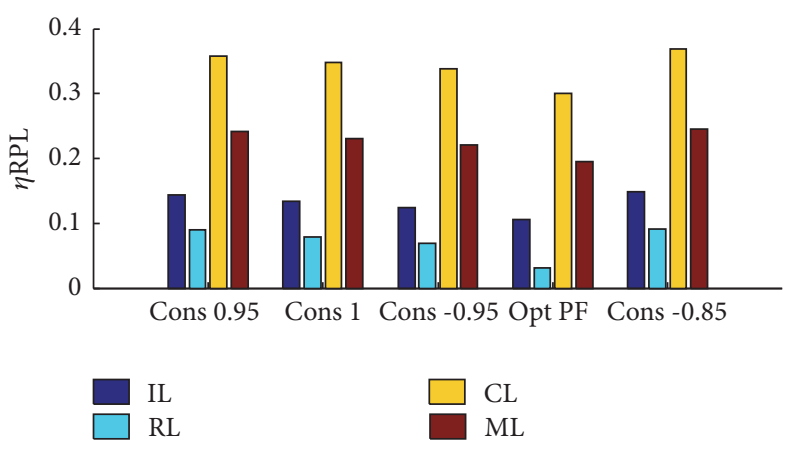

(b) RPL index

FIGURE 9: Comparative results of network loss under diverse load type conditions by using constant PFs and optimal PF regulation.

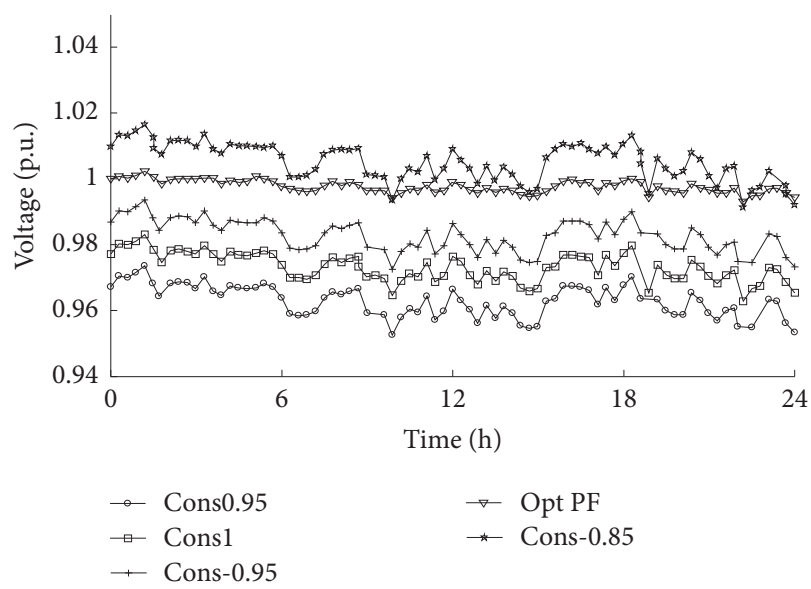

(a) Voltage profile

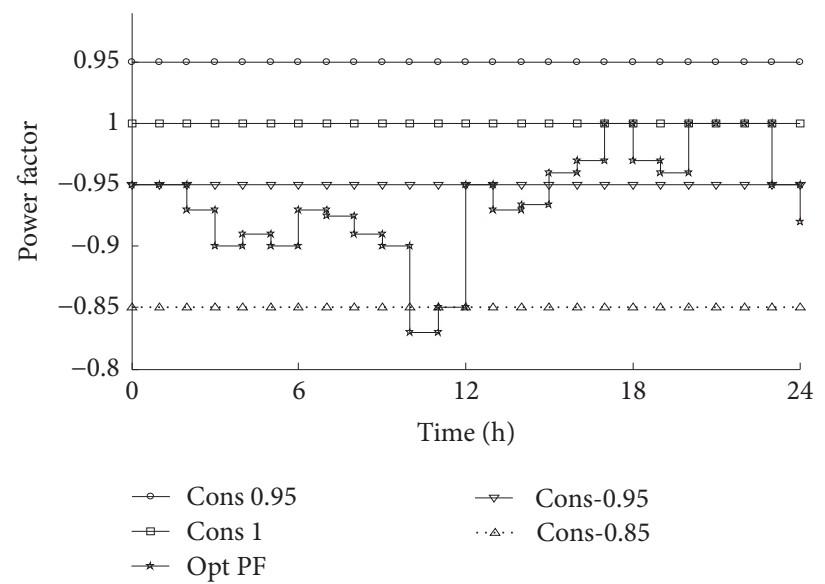

(b) Power factor

FIGURE 10: Voltage profile and power factor by using constant PFs and optimal PF regulation under mixed load condition.

TABLE 3: Computation time of different algorithms.

\begin{tabular}{lc}
\hline Algorithms & Computation time (s) \\
\hline IFA & 28.07 \\
ABC & 52.69 \\
FA & 59.87 \\
PSO & 65.53 \\
\hline
\end{tabular}

It can be known from Figure 11, compared with PSO, $\mathrm{ABC}$, and FA algorithms, that the main advantages of the IFA are the efficiency of multiobjective optimization, because it uses mainly real random numbers and it is based on the global communication among the swarming particles (the fireflies); when the iterations are around 50, the IFA method owns the minimum network loss (less than $200 \mathrm{~kW}$ ) and voltage deviation (about 0.04), while other methods need to take about 100 iterations, which means that the best results can be obtained by the proposed control method with the fewer iterations.

When best results are obtained, the computation time is counted, and the comparative results are shown in Table 3.

It can be concluded that compared with other algorithms, since IFA algorithm has less program occupation and simplified variable parameter adjustment, computation time is 28.07s, much lower than ABC (52.69s), FA (59.87s), and PSO (65.53s). Therefore, the time is much saved up by using IFA. In a word, the proposed IFA has more advantages than the existing optimal algorithms, especially in network loss, voltage deviation, and calculation time.

\section{Conclusion}

High penetration level of dispersed wind farms integration into distribution network presents great challenges and an optimal power factor regulation method based on improved firefly algorithm is proposed to ensure the instability of voltage and reduce network loss of dispersed wind farms. This paper proposes an optimal power factor regulation method under diverse load and stochastic wind conditions based on improved firefly algorithm and after discussion and verification, the conclusions are as follows:

(1) Different types of loads correspond to different characteristic of voltage profile and networks loss and the minimum networks loss and voltage deviation can be obtained based on the proposed control method.

(2) The chaos theory is introduced to improve the firefly algorithm effectively and the issues of local convergence and 


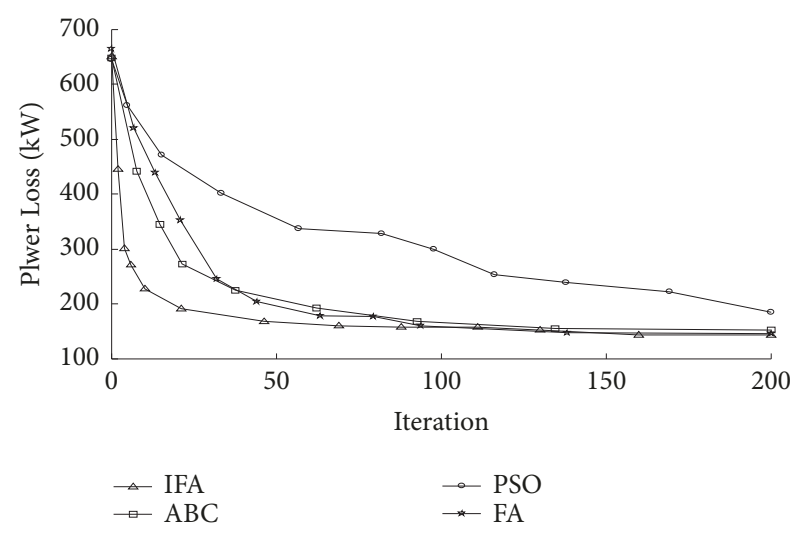

(a) Network loss

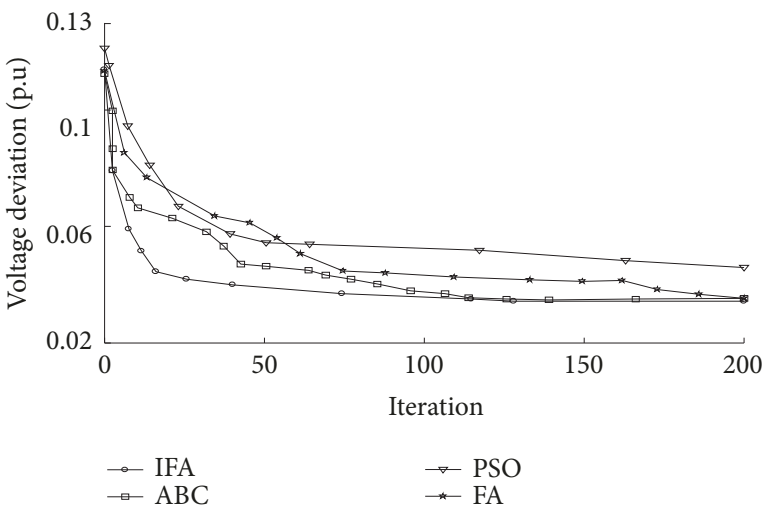

(b) Voltage deviation

FIGURE 11: Convergence curves of network loss and voltage deviation by using different algorithms under mixed load condition.

slow convergence of the original FA algorithm are well solved by IFA algorithm.

(3) Compared with the traditional constant PF control method, the proposed dynamic optimal PF control method can obtain the best results, which shows that the mutative PF can improve the stability of voltage and decrease the network loss and the network's performance can be enhanced.

\section{Data Availability}

The authors promise that all the data used in our manuscript are available and other researchers can access the data supporting the conclusions conveniently.

\section{Conflicts of Interest}

The authors declare that there are no conflicts of interest related to this paper.

\section{Acknowledgments}

This work was supported by National Natural Science Fund (51407186) of China.

\section{References}

[1] S. W. Alnaser and L. F. Ochoa, "Optimal sizing and control of energy storage in wind power-rich distribution networks," IEEE Transactions on Power Systems, vol. 31, no. 3, pp. 2004-2013, 2013.

[2] D. Suchitra, R. Jegatheesan, and T. J. Deepika, “Optimal design of hybrid power generation system and its integration in the distribution network," International Journal of Electrical Power \& Energy Systems, vol. 82, pp. 136-149, 2013.

[3] Z. Ma, Y. Liu, and Y. Shang, "Development trends of future power system and active distribution system," Proceedings of the CSEE, vol. 37, no. 1, pp. 27-35, 2017.

[4] J. Xiao, F. Gang, and R. Huang, "Total supply capability model for flexible distribution network," Proceedings of the CSEE, Automation of Electric Power Systems, vol. 41, no. 5, pp. 30-38, 2017.
[5] M. Esparza, J. Segundo, C. Nunez, X. Wang, and F. Blaabjerg, "A comprehensive design approach of power electronic-based distributed generation units focused on power-quality improvement," IEEE Transactions on Power Delivery, vol. 32, no. 2, pp. 942-950, 2017.

[6] S. Santos, D. Fitiwi, A. Bizuayehu, J. Catalao, and M. Shafiekhah, "Optimal integration of RES-based DGs with reactive power support capabilities in distribution network systems," in Proceedings of the 2016 13th International Conference on the European Energy Market (EEM), pp. 1-5, Porto, Portugal, June 2016.

[7] H. Zou, X. Han, Q. Liao, D. Liu, Z. Zhu, and W. Chen, "Penetration capacity calculation for distributed generation considering voltage quality and short circuit capacity constraints," Power System Technology, vol. 40, no. 8, pp. 2273-2280, 2016.

[8] Z. Liu and J. V. Milanović, "Probabilistic Estimation of Voltage Unbalance in MV Distribution Networks With Unbalanced Load," IEEE Transactions on Power Delivery, vol. 30, no. 2, pp. 693-703, 2015.

[9] J. Cui, J. Yang, L. Li et al., "Voltage fluctuation analysis and mitigation of distribution network containing distributed wind farm," Power System Technology, vol. 39, no. 12, pp. 3414-3420, 2015.

[10] J. Chen, T. Jiang, Y. Zhou, and Y. Li, "Reasonable allocation of distributed generation in distribution system considering voltage sag," Power System Technology, vol. 38, no. 8, pp. 22442249,2014

[11] M. Othman, W. El-Khattam, Y. Hegazy, and A. Y. Abdelaziz, "Optimal placement and sizing of voltage controlled distributed generators in unbalanced distribution networks using supervised firefly algorithm," International Journal of Electrical Power \& Energy Systems, vol. 82, pp. 105-113, 2016.

[12] X. Zhu, J. Zhang, W. Liu, and W. Qiu, "Power flow calculation of distribution system with distributed generation considering static load characteristics," Power System Technology, vol. 36, no. 2, pp. 217-223, 2012.

[13] H. Gao and J. Liu, "Coordinated planning considering different types of dg and load in active distribution network," Proceedings of the CSEE, vol. 36, no. 18, pp. 4911-4922, 2016.

[14] W. Guo, F. Liu, J. Si, D. He, R. Harley, and S. Mei, "Approximate dynamic programming based supplementary reactive power control for DFIG wind farm to enhance power system stability," Neurocomputing, vol. 170, pp. 417-427, 2015. 
[15] T. Ghennam, K. Aliouane, F. Akel, B. Francois, and E. M. Berkouk, "Advanced control system of DFIG based wind generators for reactive power production and integration in a wind farm dispatching," Energy Conversion and Management, vol. 105, pp. 240-250, 2015.

[16] J. Kim, J.-K. Seok, E. Muljadi, and Y. C. Kang, "Adaptive Q$\mathrm{V}$ scheme for the voltage control of a dfig-based wind power plant," IEEE Transactions on Power Electronics, vol. 31, no. 5, pp. 3586-3599, 2016.

[17] M. Shahzad, I. Ahmad, W. Gawlik, and P. Palensky, "Load concentration factor based analytical method for optimal placement of multiple distribution generators for loss minimization and voltage profile improvement," Energies, vol. 9, no. 287, pp. 1-21, 2016.

[18] P. Satapathy, S. Dhar, and P. K. Dash, "Stability improvement of PV-BESS diesel generator-based microgrid with a new modified harmony search-based hybrid firefly algorithm," IET Renewable Power Generation, vol. 11, no. 5, pp. 566-577, 2017.

[19] M. Huang, J. Yuan, and J. Xiao, "An adapted firefly algorithm for product development project scheduling with fuzzy activity duration," Mathematical Problems in Engineering, vol. 2015, Article ID 973291, 11 pages, 2015.

[20] Y. Wu, Q. Li, Q. Hu, and A. Borgart, "Size and topology optimization for trusses with discrete design variables by improved firefly algorithm," Mathematical Problems in Engineering, vol. 2017, Article ID 1457297, 12 pages, 2017.

[21] L. Wu, X. Yang, H. Zhou, and X. Hao, "Asymptotically optimal scenario-based multi-objective optimization for distributed generation allocation and sizing in distribution systems," International Journal of Grid and Distributed Computing, vol. 9, no. 4, pp. 75-86, 2016.

[22] P. Sivaranjania and A. Senthil Kumarb, "Hybrid Particle Swarm Optimization-Firefly algorithm (HPSOFF) for combinatorial optimization of non-slicing VLSI floorplanning," Journal of Intelligent \& Fuzzy Systems, vol. 32, pp. 661-669, 2017.

[23] X. Zhang, X. Zhang, and L. Wang, "Antenna design by an adaptive variable differential artificial bee colony algorithm," IEEE Transactions on Magnetics, vol. 50, no. 2, pp. 737-740, 2014. 


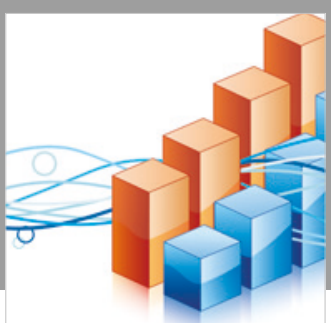

Advances in

Operations Research

\section{-n-m}
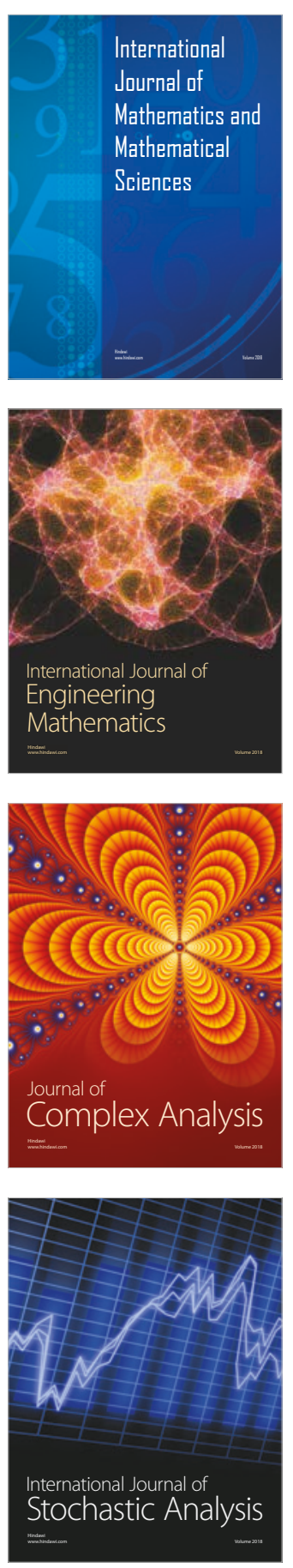
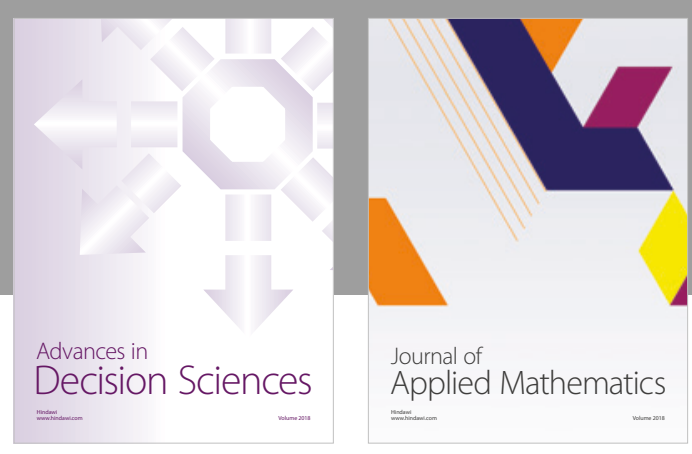

Journal of

Applied Mathematics
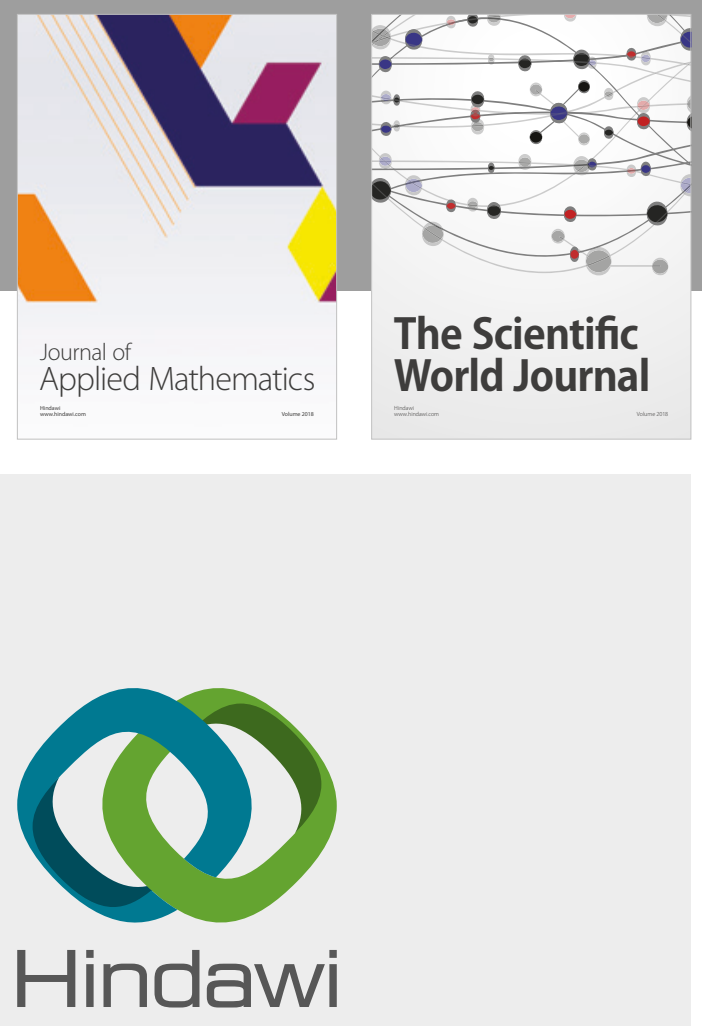

Submit your manuscripts at

www.hindawi.com

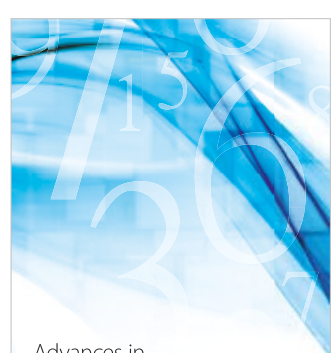

Advances in
Numerical Analysis
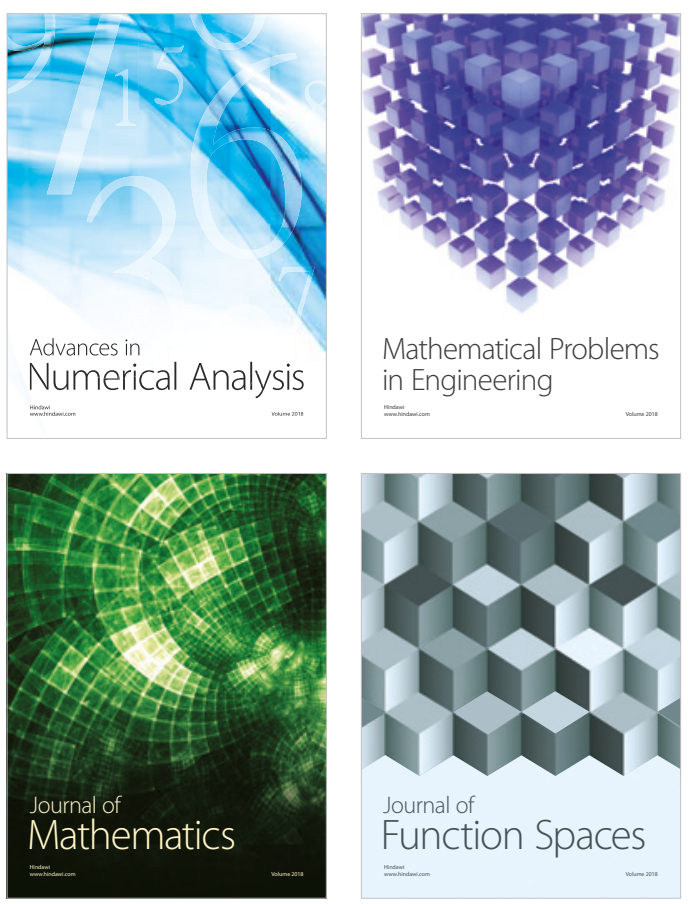

Mathematical Problems in Engineering

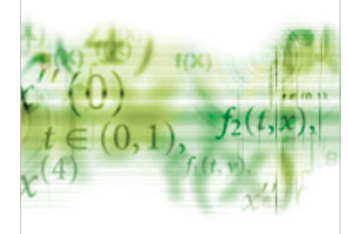

International Journal of

Differential Equations

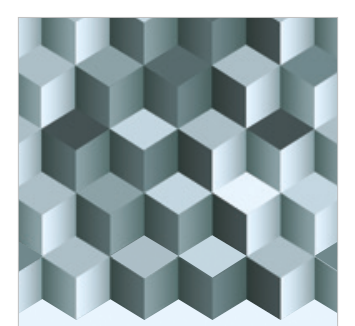

Journal of

Function Spaces

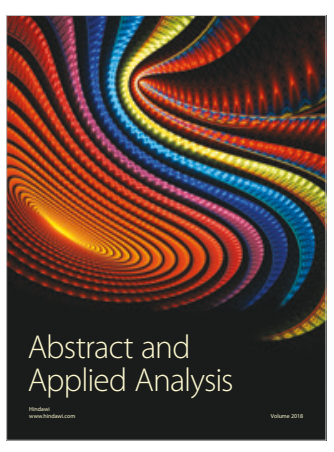

The Scientific

World Journal

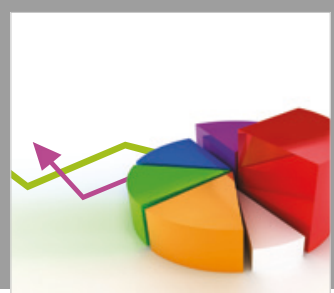

Journal of

Probability and Statistics
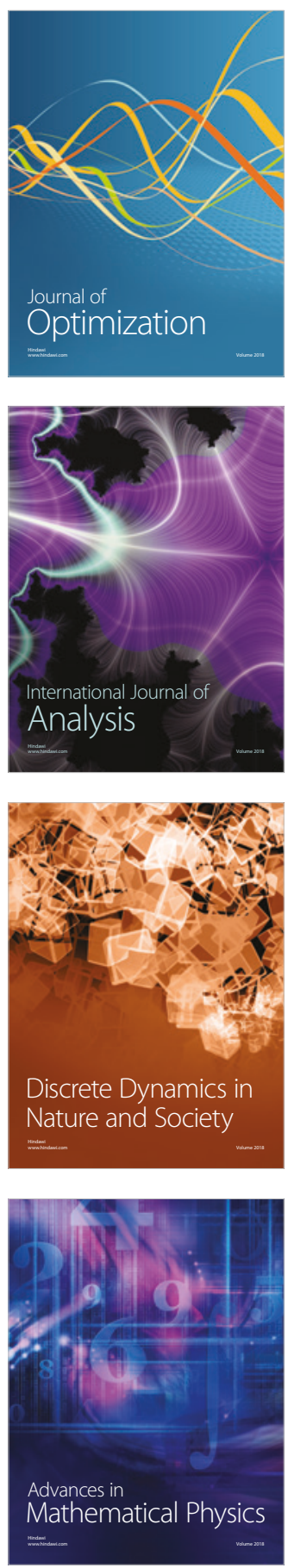